\title{
Produção e nutrição de limoeiro 'Tahiti' em função da adubação com nitrogênio e potássio em cinco safras
}

\author{
José Tadeu Alves da Silva(1), Inêz Pereira da Silva(1) e Fúlvio Rodriguez Simão(1)
} (1)Empresa de Pesquisa Agropecuária de Minas Gerais, Campo Experimental de Montes Claros, Caixa Postal 52, CEP $39400-970$ Montes
Claros, MG, Brasil. E-mail: josetadeu@epamig.br, inezps@yahoo.com.br, fulvio@epamig.br

Resumo - O objetivo deste trabalho foi estimar as doses de nitrogênio e potássio, para obter a máxima eficiência técnica e econômica e os níveis críticos destes dois nutrientes, em folhas de limão 'Tahiti' irrigado, cultivado em Neossolo Quartzarênico, em cinco safras. Utilizou-se o delineamento experimental de blocos ao acaso, com quatro repetições, em arranjo fatorial 4x5, com quatro doses de $\mathrm{N}\left(0,25,50\right.$ e $\left.100 \mathrm{~kg} \mathrm{ha}^{-1}\right)$ e cinco doses de $\mathrm{K}_{2} \mathrm{O}\left(0,25,50,100\right.$ e $\left.200 \mathrm{~kg} \mathrm{ha}^{-1}\right)$. As doses estimadas para alcançar a máxima eficiência técnica foram 90 , 99, 69, 66 e $100 \mathrm{~kg} \mathrm{ha}^{-1}$ de $\mathrm{N}$ e, para a máxima eficiência econômica, 85, 95, 67, 64 e $93 \mathrm{~kg} \mathrm{ha}^{-1}$ de N, para a primeira, a segunda, a terceira, a quarta e a quinta safra, respectivamente. As doses de $\mathrm{K}_{2} \mathrm{O}$ para obter a máxima eficiência técnica foram 126, 116, 101, 98 e $95 \mathrm{~kg} \mathrm{ha}^{-1} \mathrm{e}$, para a máxima eficiência econômica, 112, 105, 94, 90 e $88 \mathrm{~kg} \mathrm{ha}^{-1} \mathrm{de}_{2} \mathrm{O}$, para a primeira, segunda, terceira, quarta e quinta safra, respectivamente. Os níveis críticos foliares médios estimados são de $25 \mathrm{~g} \mathrm{~kg}^{-1}$ de $\mathrm{N}$ e $22 \mathrm{~g} \mathrm{~kg}^{-1}$ de K para as cinco safras.

Termos para indexação: Citrus latifolia, diagnose nutricional, estado nutricional, irrigação, nível crítico.

\section{Production and nutrition of 'Tahiti' lime as affected by nitrogen and potassium fertilization in five crop seasons}

\begin{abstract}
The objective of this work was to estimate nitrogen and potassium rates, in order to obtain maximum technical and economic efficiency and the critical levels of these two nutrients on leaves of irrigated 'Tahiti' lime tree, grown in a Typic Quartzipsamment soil in five crop seasons. A randomized complete block design was carried out with four replicates, in a $4 \times 5$ factorial arrangement, with four $\mathrm{N}$ rates $\left(0,25,50\right.$, and $\left.100 \mathrm{~kg} \mathrm{ha}^{-1}\right)$ and five $\mathrm{K}_{2} \mathrm{O}$ rates $\left(0,25,50,100\right.$, and $\left.200 \mathrm{~kg} \mathrm{ha}^{-1}\right)$. The estimated rates to achieve the maximum technical efficiency were 90, 99, 69, 66, and $100 \mathrm{~kg} \mathrm{ha}^{-1} \mathrm{~N}$, and for the maximum economic efficiency, 85, 95, 67, 64, and $93 \mathrm{~kg} \mathrm{ha}^{-1} \mathrm{~N}$, for the first, second, third, fourth, and fifth crop seasons, respectively. The rates of $\mathrm{K}_{2} \mathrm{O}$ to obtain the maximum technical efficiency were 126,116,101, 98, and $95 \mathrm{~kg} \mathrm{ha}^{-1}$, and for maximum economic efficiency, 112, 105, 94, 90, and $88 \mathrm{~kg} \mathrm{ha}^{-1} \mathrm{~K}_{2} \mathrm{O}$, respectively, for the first, second, third, fourth, and fifth crops. The average, estimated critical levels for leaf contents are $25 \mathrm{~g} \mathrm{~kg}^{-1} \mathrm{~N}$ and $22 \mathrm{~g} \mathrm{~kg}^{-1} \mathrm{~K}$, in five crop seasons.

Index terms: Citrus latifolia, nutritional diagnosis, nutritional status, irrigation, critical level.
\end{abstract}

\section{Introdução}

A elevação da produtividade de plantas cítricas depende, em grande parte, da aplicação do $\mathrm{N}$ e $\mathrm{K}$ (Alva et al., 2006). Quaggio et al. (2011) verificaram que os rendimentos máximos de frutos de laranja 'Pêra' e 'Valência' foram obtidos com aplicações de 242 e $302 \mathrm{~kg} \mathrm{ha}^{-1}$ de $\mathrm{K}_{2} \mathrm{O}$, respectivamente, aplicados a solo de textura média, com baixo teor de $\mathrm{K}(0,06$ $\mathrm{cmol}_{\mathrm{c}} \mathrm{dm}^{-3}$ ). Penna et al. (2012) verificaram que a aplicação de $\mathrm{N}, \mathrm{P}$ e K proporcionaram elevação da altura e da massa de matéria seca de mudas de limoeiro 'Tahiti' enxertado em 'Flying Dragon'.

Quaggio et al. (1996) recomendam para citros cultivados em solo com teor de $\mathrm{K}$ abaixo de 0,08 $\mathrm{cmol}_{\mathrm{c}} \mathrm{dm}^{-3}$, as seguintes doses de $\mathrm{N}_{\text {e }} \mathrm{K}_{2} \mathrm{O}: 20$ e 5,40 e 20,50 e 38,75 e 50,100 e $75 \mathrm{~kg} \mathrm{ha}^{-1}$, respectivamente, para o primeiro, segundo, terceiro, quarto e quinto anos de idade. No entanto, estas são recomendações para citros em geral, que não especificam a espécie e nem a variedade.

Acredita-se que pode haver discrepância entre esses valores e aqueles obtidos especificamente para o limão 'Tahiti', cuja espécie é Citrus latifolia Tanaka, diferentemente da laranjeira, que é Citrus sinensis Osbeck.

No Brasil, há poucas publicações de pesquisas sobre adubação do limoeiro 'Tahiti' com N e K. Assim, as recomendações para aplicações desses dois nutrientes para essa cultura são baseadas, em sua maioria, em 
dados obtidos em experimentos com a cultura da laranjeira.

As principais ferramentas para verificar as necessidades nutricionais de plantas cítricas são os resultados da análise de solo e de folhas. Para realizar a diagnose nutricional pela análise foliar, é preciso estabelecer os níveis críticos dos nutrientes a serem diagnosticados.

O objetivo deste trabalho foi estimar as doses de nitrogênio e potássio, para obter a máxima eficiência técnica e econômica e os níveis críticos destes dois nutrientes, em folhas de limão 'Tahiti' irrigado, cultivado em Neossolo Quartzarênico, em cinco safras.

\section{Material e Métodos}

O experimento foi realizado no Município de Jaíba, localizado na região semiárida do norte de Minas Gerais, Brasil $\left(15^{\circ} 48^{\prime} 09^{\prime \prime} \mathrm{S}, 43^{\circ} 18^{\prime} 32^{\prime \prime} \mathrm{W}\right.$, à altitude de $533 \mathrm{~m}$ ). As médias anuais de precipitação foram de $800 \mathrm{~mm}$, a temperatura foi de $25^{\circ} \mathrm{C}$ e a umidade relativa do ar de $56 \%$. Essa região apresenta clima do tipo Aw, segundo a classificação de Köppen-Geiger.

O solo da área experimental é classificado como Neossolo Quartzarênico (Sistema..., 1999) e, no período do experimento, apresentava as seguintes características, na camada 0-0,20 m: $\mathrm{pH}$ em água, 6,6; Al trocável, $0,0 \mathrm{cmol}_{\mathrm{c}} \mathrm{dm}^{-3} ; \mathrm{H}+\mathrm{Al}, 1,1 \mathrm{cmol}_{\mathrm{c}} \mathrm{dm}^{-3} ; \mathrm{Ca}$, 1,6 $\mathrm{cmol}_{\mathrm{c}} \mathrm{dm}^{-3} ; \mathrm{Mg}, 0,6 \mathrm{cmol}_{\mathrm{c}} \mathrm{dm}^{-3} ; \mathrm{T}, 3,3 \mathrm{cmol}_{\mathrm{c}} \mathrm{dm}^{-3}$; V, 68\%; P-Mehlich 1, 8,7 mg dm d $^{-3}$ K-Mehlich 1, $29 \mathrm{mg} \mathrm{dm}^{-3}$; matéria orgânica, $0,6 \mathrm{dag} \mathrm{kg}^{-1}$; areia, 850 $\mathrm{g} \mathrm{kg}^{-1}$; argila, $100 \mathrm{~g} \mathrm{~kg}^{-1}$; e silte, $50 \mathrm{~g} \mathrm{~kg}^{-1}$.

$\mathrm{O}$ experimento foi realizado em pomar de limoeiro 'Tahiti' (Citrus latifolia Tanaka) com quatorze meses de idade, enxertado sobre limoeiro 'Cravo' (Citrus limonia $\mathrm{L}$. Osbeck). O plantio das mudas foi realizado em janeiro de 2008 , em covas de $0,40 \times 0,40 \times 0,40 \mathrm{~m}$, no espaçamento de $8 \mathrm{~m}$ entre fileiras e $5 \mathrm{~m}$ entre plantas. A adubação de plantio foi baseada nas recomendações de Quaggio et al. (1996), com aplicações de $120 \mathrm{~g}$ de $\mathrm{P}_{2} \mathrm{O}_{5}\left(30 \mathrm{~kg} \mathrm{ha}^{-1}\right.$ de $\left.\mathrm{P}_{2} \mathrm{O}_{5}\right)$ e $15 \mathrm{~L}$ por cova de esterco bovino curtido, com teor de $\mathrm{Ne} \mathrm{K}$ de 1,2 e $0,52 \mathrm{dag}_{\mathrm{kg}}{ }^{-1}$, respectivamente. A adubação fosfatada foi aplicada anualmente $\left(30 \mathrm{~kg} \mathrm{ha}^{-1}\right.$ de $\mathrm{P}_{2} \mathrm{O}_{5}$ ), tendo-se utilizado o superfosfato simples $\left(18 \% \mathrm{P}_{2} \mathrm{O}_{5}, 20 \% \mathrm{Ca}, 12 \% \mathrm{~S}\right)$. Os micronutrientes foram aplicados à superfície do solo na projeção da copa da planta, quatro meses após o plantio, com a utilização de $50 \mathrm{~g}$ de FTE BR-12 por planta $\left(12,5 \mathrm{~kg} \mathrm{ha}^{-1}\right)(\mathrm{S}, 3,9 \%$; B, 1,8\%; Mn, 2,0\% e $\mathrm{Zn}, 9,0 \%$ ); essa dose foi repetida anualmente. Não houve necessidade de realizar a calagem.

O delineamento experimental em blocos ao acaso foi conduzido com quatro repetições, em arranjo fatorial $4 \times 5$, correspondente a quatro doses de $\mathrm{N}(0,25,50$ e $100 \mathrm{~kg} \mathrm{ha}^{-1}$ por ano) e cinco doses de $\mathrm{K}_{2} \mathrm{O}(0,25,50$, 100 e $200 \mathrm{~kg} \mathrm{ha}^{-1}$ por ano), no total de 20 tratamentos e 80 unidades experimentais. As aplicações do $\mathrm{N} \mathrm{e} \mathrm{K}_{2} \mathrm{O}$ foram realizadas mensalmente e se iniciaram quatorze meses após o plantio das mudas, aplicadas à superfície do solo, na projeção da copa da planta. As fontes de $\mathrm{N}$ e $\mathrm{K}_{2} \mathrm{O}$ foram ureia e cloreto de potássio $(\mathrm{KCl})$, respectivamente.

Cada parcela do ensaio constituiu-se de quatro plantas, e a parcela útil, das duas plantas centrais. $\mathrm{O}$ experimento foi irrigado por sistema de microaspersão, com manejo da lâmina de água por meio de medidas da evaporação do tanque classe A.

Amostras de folhas foram coletadas na primeira, terceira e quinta safra, em ramos com frutos de diâmetro em torno de $2 \mathrm{~cm}$, gerados na primavera, com aproximadamente 6-7 meses de idade. Retirou-se a 3. ${ }^{\text {a }}$ folha a partir do fruto e duas folhas em cada ponto cardeal, no total de oito folhas por planta (Quaggio et al., 1996). Nas amostras de folhas, determinaram-se os teores de $\mathrm{N}, \mathrm{P}, \mathrm{K}, \mathrm{S}, \mathrm{Ca}, \mathrm{Mg}, \mathrm{B}, \mathrm{Cu}, \mathrm{Fe}, \mathrm{Mn}$ e $\mathrm{Zn}$, conforme descrito em Malavolta et al.(1997).

Dois anos após o plantio do limoeiro, iniciaramse as colheitas de limão, em cada planta útil do experimento. Os frutos de limão colhidos foram pesados para a determinação da produção de cada tratamento. A primeira safra colhida foi em 2010, e a quinta em 2014. As colheitas de cada safra foram realizadas quatro vezes ao ano (janeiro, maio, agosto e dezembro), quando os frutos apresentaram coloração verde-escura e casca sem rugosidade.

Os dados obtidos foram submetidos à análise de variância (Teste F) e ajustes de regressão, e tendose selecionado aqueles com significância mínima de $5 \%$ de probabilidade, com maior coeficiente de determinação $\left(\mathrm{R}^{2}\right)$.

As doses de $\mathrm{N}$ e $\mathrm{K}_{2} \mathrm{O}$ para as máximas eficiências técnica e econômica foram estimadas conforme descrito em Alvarez V. (1994). Os preços do $\mathrm{N}$ e $\mathrm{K}_{2} \mathrm{O}$ contidos na ureia e no $\mathrm{KCl}$, respectivamente, foram: $\mathrm{R} \$ 3,64 \mathrm{~kg}^{-1}$ de $\mathrm{N}$ e R $\$ 3,10 \mathrm{~kg}^{-1}$ de $\mathrm{K}_{2} \mathrm{O}$ (cotados no comércio do norte de Minas Gerais, em setembro de 
2015). O preço do quilograma de limão 'Tahiti' foi R\$ 0,50 (Associação Central dos Fruticultores do Norte de Minas, 2015).

\section{Resultados e Discussão}

Não houve interação significativa entre $\mathrm{N}$ e $\mathrm{K}_{2} \mathrm{O}$ quanto à produção do limoeiro 'Tahiti'. A produção do limão aumentou de forma quadrática com as doses de $\mathrm{N}$ aplicadas ao solo, nas cinco safras do limoeiro (Figura 1). As doses estimadas para alcançar a máxima eficiência técnica foram 90, 99, 69, 66 e $100 \mathrm{~kg} \mathrm{ha}^{-1}$ de $\mathrm{N}$, na primeira, segunda, terceira, quarta e quinta safra, respectivamente. As doses para obter a máxima eficiência econômica foram 85, 95, 67, 64 e $93 \mathrm{~kg} \mathrm{ha}^{-1}$ de $\mathrm{N}$ com a produtividade de 6,7, 13,0, 17,5, 18,4 e $27,0 \mathrm{Mg} \mathrm{ha}^{-1}$ na primeira, segunda, terceira, quarta e quinta safra, respectivamente. Quaggio et al. (1996) recomenda as seguintes doses: 20, 40, 50, 75 e $100 \mathrm{~kg} \mathrm{ha}^{-1}$ de $\mathrm{N}$ para o primeiro, segundo, terceiro, quarto e quinto anos de idade, respectivamente, para uma população de 250 plantas por hectare. No presente trabalho, o limoeiro tinha dois anos de idade na primeira safra e seis anos na quinta safra. Portanto, verificou-se que as doses econômicas de $\mathrm{N}$ obtidas na primeira e segunda safras foram superiores àquelas recomendadas

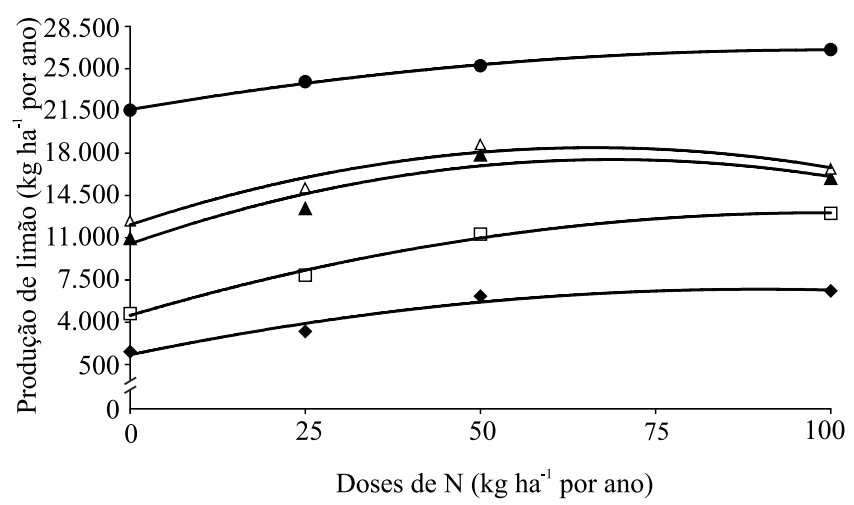

$\begin{array}{ll}\text { - Primeira safra }=\hat{y}=1290+120,8 x-0,671^{* *} x^{2} & R^{2}=0,956 \\ \text { 口Segunda safra }=\hat{y}=4543+171,6 x-0,865^{* *} x^{2} & R^{2}=0,993 \\ \Delta \text { Terceira safra }=\hat{y}=10467+202,9 x-1,471^{* *} x^{2} & R^{2}=0,906 \\ \Delta \text { Quarta safra }=\hat{y}=12050+193,0 x-1,456^{* *} x^{2} & R^{2}=0,940 \\ \text {-Quinta safra }=\hat{y}=21597+99,1,0 x-0,497 * * x^{2} & R^{2}=0,998\end{array}$

Figura 1. Produção de limoeiro 'Tahiti', em função da adubação com $\mathrm{N}$, em cinco safras. ${ }^{* *}$ Significativo a $1 \%$ de probabilidade. por Quaggio et al. (1996); da terceira à quinta safra, as doses foram próximas do recomendado por esses autores. Fidalski \& Auler (2007) obtiveram a máxima eficiência técnica da laranjeira 'Valência' com a dose de $124 \mathrm{~kg} \mathrm{ha}^{-1}$ de $\mathrm{N}$, aplicada a solo de textura arenosa, no sexto ano após o plantio, para uma população de 345 plantas por hectare. Se considerarmos essa mesma população de plantas para o limão 'Tahiti', a dose para obter a máxima eficiência técnica de limão, na quinta safra, será de $138 \mathrm{~kg} \mathrm{ha}^{-1}$ de $\mathrm{N}$, levemente superior à obtida pelos autores supramencionados.

Esperava-se que a dose de $\mathrm{N}$ para obter a produção econômica máxima, nas duas primeiras safras, fosse menor do que a dose aplicada na quarta e quinta safras, porque o limoeiro apresentou produção cerca de cinco vezes maior na quinta safra, em comparação à obtida na primeira safra, cuja produção máxima econômica, com aplicação de $85 \mathrm{~kg} \mathrm{ha}^{-1}$ de N, aumentou $420 \%$ em comparação à produção sem aplicação de $\mathrm{N}$. Na quinta safra, o aumento da produção foi de apenas $23 \%$, com aplicação de $93 \mathrm{~kg} \mathrm{ha}^{-1}$ de N, ou seja, o limoeiro respondeu à aplicação de $\mathrm{N}$ com maior intensidade na primeira safra. Este fato pode ser atribuído ao menor desenvolvimento do sistema radicular da planta na primeira safra, que explorou uma área menor para absorção de N, pois, Magalhães Filho (2008) verificou que o comprimento total do sistema radicular da laranjeira 'Valência' irrigada, enxertada em limão 'Cravo', aumentou de $30 \mathrm{~cm}$, na medida inicial, para $70 \mathrm{~cm}$ na medida realizada três meses depois, o que mostra que as raízes dos citros apresentam crescimento significativo, à medida que avançam em idade.

Pelos resultados obtidos, na quinta safra, por Magalhães Filho (2008), o sistema radicular, provavelmente, já estava mais desenvolvido e alcançou maior profundidade no solo, o que favoreceu a exploração de maior área e reduziu as perdas de $\mathrm{N}$ pela lixiviação, que são grandes em solo muito arenoso sob irrigação (Mendes et al., 2015). Souza et al., (2007) estudaram o sistema radicular da laranjeira 'Pera' de quatorze anos, irrigada, enxertada em limoeiro 'Rugoso', e encontraram raízes até a profundidade de 1,2 m. Na laranjeira 'Valência' irrigada, enxertada em limoeiro 'Cravo', com 36 anos de idade, Testezlaf et al. (2007) encontraram massa de raízes a 2,20 m. Esse crescimento do sistema radicular em profundidade possibilita ao limoeiro também absorver maior quantidade de $\mathrm{N}$, proveniente de camadas profundas 
do solo (Neves et al., 2004), mesmo sem aplicação desse nutriente, e aumentar a eficiência de absorção da planta, reduzindo, assim, o ganho de produção com a aplicação de N.

A produção do limoeiro 'Tahiti' aumentou de forma quadrática com as doses de $\mathrm{K}_{2} \mathrm{O}$ aplicadas no solo (Figura 2). As doses para obter a máxima eficiência técnica foram 126, 116, 101, 98 e $95 \mathrm{~kg} \mathrm{ha}^{-1}$ de $\mathrm{K}_{2} \mathrm{O}$, enquanto as doses para obter a máxima eficiência econômica foram 112, 105, 94, 90 e $88 \mathrm{~kg} \mathrm{ha}^{-1}$ de $_{2} \mathrm{O}$. A máxima eficiência econômica obtida por limoeiro foram 6,$3 ; 11,9 ; 17,9 ; 18,3$ e $27,0 \mathrm{~kg} \mathrm{ha}^{-1}$ na primeira, segunda, terceira, quarta e quinta safra, respectivamente. As doses de $\mathrm{K}_{2} \mathrm{O}$ para obter a máximas eficiência técnica e econômica de limão reduziram-se da primeira para a quinta safra. Semelhantemente ao que foi discutido sobre o $\mathrm{N}$, o sistema radicular do limoeiro, possivelmente, desenvolveu-se e alcançou maior profundidade no solo na quinta safra, em comparação à primeira safra (Souza et al., 2007; Magalhães Filho, 2008), elevou a eficiência da planta em absorver os nutrientes, reduziu as perdas de $\mathrm{K}$ por lixiviação e possibilitou a absorção de maior quantidade do $\mathrm{K}_{2} \mathrm{O}$ existente em camadas mais profundas do solo (Duarte et al., 2013).

Em pomar cultivado em solo com teor de $\mathrm{K}$ menor do que $0,7 \mathrm{mmol}_{\mathrm{c}} \mathrm{dm}^{3}\left(29 \mathrm{mg} \mathrm{dm}^{-3}\right)$, no espaçamento $8 \times 5 \mathrm{~m}$, as doses de $\mathrm{K}_{2} \mathrm{O}$ recomendadas por Quaggio

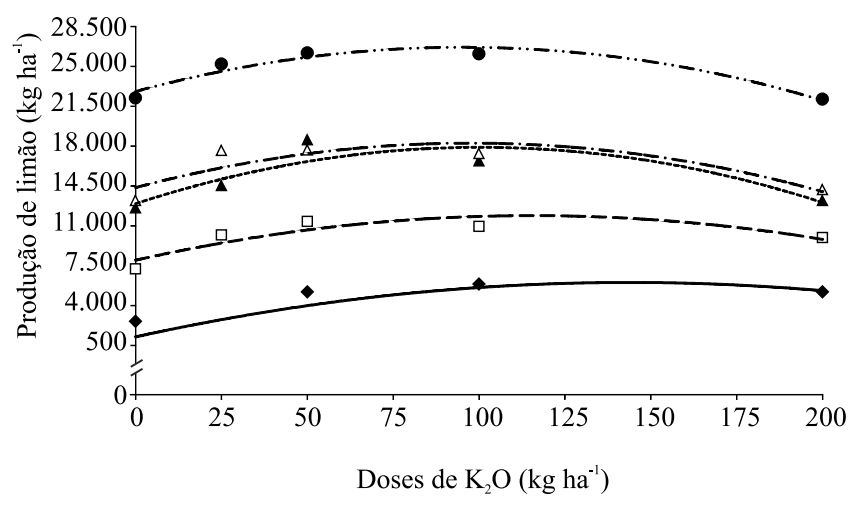

$$
\begin{aligned}
& \text { - - Primeira safra }=\hat{y}=2924+53,5 x-0,212 * * x^{2} \quad R^{2}=0,940 \\
& \text { 口 - - - - Segunda safra }=\hat{y}=7974+67,76 x-0,293 * * x^{2} \quad R^{2}=0,771 \\
& \Delta \text {..........Terceira safra }=\hat{y}=12929+98,7 x-0,491 * * x^{2} \quad R^{2}=0,775 \\
& \Delta \text {-.... Quarta safra }=\hat{y}=14349+80,0 x-0,409 * * x^{2} \quad R^{2}=0,749 \\
& \text { - -...... Quinta safra }=\hat{y}=22789+81,5 x-0,427 * * x^{2} \quad R^{2}=0,928
\end{aligned}
$$

Figura 2. Produção de limoeiro 'Tahiti', em função da adubação com $\mathrm{K}$ em cinco safras. ${ }^{* *}$ Significativo a $1 \%$ de probabilidade. et al. (1996) são 5, 20, 38, 50 e $75 \mathrm{~kg} \mathrm{ha}^{-1}$, para o primeiro, segundo, terceiro, quarto e quinto anos de idade, respectivamente. Essas doses recomendadas aumentam com a idade da planta, ao contrário do ocorrido no presente trabalho, em que as doses de $\mathrm{K}_{2} \mathrm{O}$ apresentaram valores maiores na primeira safra e foram se reduzindo até a quinta safra.

Considerando-se que, na primeira e na quarta safras, as plantas de limão 'Tahiti' estavam com dois e cinco anos, respectivamente, as doses de $\mathrm{K}_{2} \mathrm{O}$, recomendadas para esses estágios, seriam, respectivamente, 20 e $75 \mathrm{~kg} \mathrm{ha}^{-1}$ (Quaggio et al., 1996), portanto, menores do que 112 e $90 \mathrm{~kg} \mathrm{ha}^{-1}$, obtidas no presente trabalho. Na quinta safra, a planta de limão 'Tahiti' estava com seis anos de idade e alcançou produção máxima econômica de $27 \mathrm{Mg} \mathrm{ha}^{-1}$, com dose de $88 \mathrm{~kg} \mathrm{ha}^{-1}$ de $\mathrm{K}_{2} \mathrm{O}$, próxima de $90 \mathrm{~kg} \mathrm{ha}^{-1}$ de $\mathrm{K}_{2} \mathrm{O}$ recomendada por Quaggio et al. (1996). Estes autores, em Quaggio et al. (2011), estimaram as doses econômicas de 200 e $270 \mathrm{~kg} \mathrm{ha}^{-1}$ de $\mathrm{K}_{2} \mathrm{O}$, para a obtenção de produções máximas econômicas de 46 e $49 \mathrm{Mg} \mathrm{ha}^{-1}$ de laranjeira 'Pera' e 'Valência', respectivamente; tal produção é quase duas vezes maior do que que a obtida com o limão 'Tahiti' (27 $\mathrm{Mg} \mathrm{ha}^{-1}$ ), que também exigiu doses de $\mathrm{K}_{2} \mathrm{O}$ duas vezes e meia maiores. Essas produções maiores podem ser explicadas pelas diferenças de idade das plantas de limão 'Tahiti', com seis anos na quinta safra, e as de laranjeiras 'Pera' e 'Valência', com dez anos. O potencial de produção dos citros se eleva com a sua idade, como pode ser constatado no trabalho de Auler et al. (2008) e no presente trabalho.

Não houve interação significativa entre $\mathrm{N}$ e $\mathrm{K}_{2} \mathrm{O}$ para os teores foliares de nutrientes (Tabela 1). Apenas os teores de $\mathrm{N}, \mathrm{K}, \mathrm{Ca}, \mathrm{Mg}$ e $\mathrm{Mn}$ na folha foram influenciados significativamente pela aplicação de $\mathrm{N}$. $\mathrm{O}$ teor de $\mathrm{N}$ foliar elevou-se linearmente com as doses de $\mathrm{N}$ aplicadas ao solo. Substituindo-se as doses de $\mathrm{N}$ que proporcionaram a máxima eficiência técnica, estimaram-se os níveis críticos foliares de $\mathrm{N}$, que foram 27,23 e $24 \mathrm{~g} \mathrm{~kg}^{-1}$ na primeira, terceira e quinta safra, respectivamente, em que o nível crítico médio de $\mathrm{N}$ foi $25 \mathrm{~g} \mathrm{~kg}^{-1}$. Verificou-se que o nível crítico de $\mathrm{N}$ foi maior na primeira do que na terceira e quinta safras, o que pode estar relacionado ao efeito de diluição. Auler et al. (2008) verificaram que, em quatro anos, o volume de copa da laranjeira 'Valência', enxertada em limão 'Cravo', elevou-se de $16,7 \mathrm{~m}^{3}$ para $23,7 \mathrm{~m}^{3}$, o que possivelmente ocorreu no presente trabalho com o limão 'Tahiti'. Teores de 23 a $27 \mathrm{~g} \mathrm{~kg}^{-1}$ de N são 
considerados adequados em folhas dos citros (Quaggio et al., 1996). O valor de nível crítico obtido para o limão 'Tahiti' se encontra nesse intervalo e próximo do estimado por Fidalski \& Auler (2007), em folhas da laranjeira 'Valência', que foi de $26 \mathrm{~g} \mathrm{~kg}^{-1}$.

$\mathrm{O}$ teor de $\mathrm{K}$ foliar reduziu-se linearmente em função do aumento das doses de $\mathrm{N}$ na primeira, terceira e quinta safras (Tabela 1). A interação mais comum relacionada ao $\mathrm{N}$ é a que ocorre com o $\mathrm{K}$. Esses são os dois nutrientes minerais absorvidos em maior quantidade pela maioria das plantas, e as interações entre ambos, normalmente, são do tipo não competitivo (Cantarella, 2007). A absorção de um nutriente eleva a demanda pelo outro. Assim, o estímulo do crescimento provocado pela adição de $\mathrm{N}$ pode levar à deficiência de $\mathrm{K}$, por efeito de diluição (Cantarella, 2007).

Os teores de $\mathrm{Ca}$ e $\mathrm{Mg}$ em folhas de limoeiro 'Tahiti' aumentaram linearmente com o aumento das doses de $\mathrm{N}$ (Tabela 1). Este fato pode ser atribuído à redução do teor de $\mathrm{K}$ foliar, causada pelas doses de $\mathrm{N}$, que, por sua vez, favoreceram a elevação dos teores de Ca e $\mathrm{Mg}$ (Cantarella, 2007). O teor de Ca foliar na primeira, terceira e quinta safras variou de 33,0 a $40,0 \mathrm{~g} \mathrm{~kg}^{-1}$, e o de $\mathrm{Mg}$ de 2,1 a 3,4 $\mathrm{g} \mathrm{kg}^{-1}$, tanto na ausência quanto com a dose de $\mathrm{N}$ que proporcionou a máxima eficiência econômica, respectivamente, o que indica que, com as aplicações das doses de $\mathrm{N}$ que proporcionaram a máxima eficiência econômica do limoeiro, os teores de $\mathrm{Ca}$ e $\mathrm{Mg}$ estiveram dentro da faixa considerada adequada (Quaggio et al., 1996), que é de 35 a $45 \mathrm{~g} \mathrm{~kg}^{-1}$ e de 2,5 a 4,0 $\mathrm{g} \mathrm{kg}^{-1}$ para $\mathrm{Ca}$ e $\mathrm{Mg}$, respectivamente.

Entre os micronutrientes, apenas o teor foliar de $\mathrm{Mn}$ foi influenciado pelas doses de N. O teor de Mn elevouse linearmente com a aplicação de $\mathrm{N}$ e alcançou teores de 125,121 e $98 \mathrm{mg} \mathrm{kg}^{-1}$, na primeira, terceira e quinta safra, respectivamente, com aplicações das doses de $\mathrm{N}$ que proporcionaram a máxima eficiência econômica. Esses valores são maiores do que os considerados adequados, que variam de 35 a $50 \mathrm{mg} \mathrm{kg}^{-1}$ (Quaggio et al., 1996). As concreções ferro-manganosas são comuns em solos no norte de Minas Gerais (Oliveira et al., 2001), o que favorece o alto teor de Mn total nesses solos e, possivelmente, são a causa do alto teor de $\mathrm{Mn}$ em folhas de limoeiro. A fonte de $\mathrm{N}$ na forma de ureia reduz o $\mathrm{pH}$ do solo, que, por sua vez, aumenta a disponibilidade do Mn (Souza et al., 2010).

Entre os nutrientes nas folhas de limoeiro, apenas K, $\mathrm{Ca}$ e $\mathrm{Mg}$ foram influenciados significativamente pelas doses de $\mathrm{K}_{2} \mathrm{O}$ aplicadas ao solo. $\mathrm{O}$ teor de $\mathrm{K}$ foliar elevou-se linearmente com as aplicações das doses de $\mathrm{K}_{2} \mathrm{O}$ no solo, na primeira, terceira e quinta safras.

Substituindo-se as doses de $\mathrm{K}_{2} \mathrm{O}$ que proporcionaram a máxima eficiência técnica de limão na primeira, terceira e quinta safras, nas regressões ajustadas entre os teores de $\mathrm{K}$ foliar em função de doses de $\mathrm{K}_{2} \mathrm{O}$, estimaram-se os níveis críticos de $\mathrm{K}$ nas folhas de limoeiro na primeira, terceira e quinta safra, que foram 22,22 e $21 \mathrm{~g} \mathrm{~kg}^{-1}$, respectivamente, e apresentaram nível crítico médio de $\mathrm{K}$ de $22 \mathrm{~g} \mathrm{~kg}^{-1}$, valor acima do teor considerado adequado para os citros, que varia de 10 a $15 \mathrm{~g} \mathrm{~kg}^{-1}$ (Quaggio et al., 1996). Mattos Junior et al. (2010) verificaram que os teores foliares de K, avaliados em onze pomares de limão 'Tahiti' no Estado de São Paulo, variaram de 11 a $17 \mathrm{~g} \mathrm{~kg}^{-1}$, em razão da grande variabilidade das características químicas dos solos onde o limão 'Tahiti' foi cultivado. Os níveis críticos estimados por Quaggio et al. (2011) para a laranjeira 'Pera' e 'Valência' foram 13 e $15 \mathrm{~g} \mathrm{~kg}^{-1}$, respectivamente.

Tabela 1. Regressões ajustadas entre os teores de N, K, Ca, MG e Mn em folhas de limão Tahiti, em função de doses de N e $\mathrm{K}_{2} \mathrm{O}$ aplicadas a Neossolo Quartzarênico no primeiro, segundo, terceiro, quarto e quinto anos de produção.

\begin{tabular}{|c|c|c|c|c|c|c|c|c|}
\hline \multirow{2}{*}{ Doses } & $\mathrm{N}$ & \multicolumn{2}{|l|}{$\mathrm{K}$} & \multicolumn{2}{|l|}{$\mathrm{Ca}$} & \multicolumn{2}{|l|}{$\mathrm{Mg}$} & $\mathrm{Mn}$ \\
\hline & Equação & Equação & $\mathrm{R}^{2}$ & Equação & $\mathrm{R}^{2}$ & Equação & $\mathrm{R}^{2}$ & Equação \\
\hline & \multicolumn{8}{|c|}{ Primeira safra } \\
\hline $\mathrm{N}$ & $\hat{\mathrm{y}}=25,9+0,015 * * x 0,987$ & $\hat{\mathrm{y}}=22,4-0,059 * * \mathrm{x}$ & 0,963 & $\hat{y}=36,8+0,033 * x$ & 0,938 & $\hat{y}=2,1+0,006^{*} x$ & 0,966 & $\hat{y}=32,2+1,030 * * x \quad 0,975$ \\
\hline$\underline{K}$ & $\hat{\mathrm{y}}=\mathrm{ns}$ & $\hat{y}=15,6+0,054 * * x$ & 0,906 & $\hat{y}=43,7-0,077 * x$ & 0,956 & $\hat{y}=2,8-0,006^{*} x$ & 0,871 & $\hat{\mathrm{y}}={ }^{\mathrm{ns}}$ \\
\hline & \multicolumn{8}{|c|}{ Terceira safra } \\
\hline $\mathrm{N}$ & $\hat{\mathrm{y}}=20,1+0,043 * * x 0,954$ & $\hat{\mathrm{y}}=23,6-0,059 * *_{\mathrm{x}}$ & 0,991 & $\hat{y}=35,5+0,046^{*} x$ & 0,994 & $\hat{y}=2,9+0,005^{*} x$ & 0,878 & $\hat{y}=50,7+1,020 * * x \quad 0,998$ \\
\hline$\underline{K}$ & $\hat{y}=n s$ & $\hat{y}=17,6+0,044 * * x$ & 0,909 & $\hat{\mathrm{y}}=42,8-0,071 *_{\mathrm{x}}$ & 0,962 & $\hat{y}=3,3-0,004 * x$ & 0,933 & $\hat{y}={ }^{n s}$ \\
\hline & \multicolumn{8}{|c|}{ Quinta safra } \\
\hline $\mathrm{N}$ & $\hat{\mathrm{y}}=20,8+0,030 * * x 0,967$ & $\hat{\mathrm{y}}=21,1-0,025 * * \mathrm{x}$ & 0,988 & $\hat{y}=33,0+0,026^{*} x$ & 0,981 & $\hat{y}=3,0+0,003 * x$ & 0,966 & $\hat{y}=48,8+0,490 * * x \quad 0,995$ \\
\hline $\mathrm{K}$ & $\hat{\mathrm{y}}=\mathrm{ns}$ & $\hat{y}=18,1+0,030 * *_{x}$ & 0,952 & $\hat{y}=37,9-0,049 * x$ & 0,940 & $\hat{\mathrm{y}}=3,3-0,003 * \mathrm{x}$ & 0,800 & $\hat{y}=n s$ \\
\hline
\end{tabular}

* e**Significativo a 5 e $1 \%$ de probabilidade. 
Dias et al. (2013) estimaram nível crítico de K foliar de $7,0 \mathrm{~g} \mathrm{~kg}^{-1}$ para laranjeira 'Pera' cultivada na Amazônia Central. Para Santana et al. (2008), as discrepâncias entre esses padrões nutricionais estão relacionadas às diferenças das condições edafoclimáticas dos locais de onde eles foram obtidos. Portanto, é importante que os padrões nutricionais dos nutrientes foliares sejam estabelecidos para cada região produtora.

Os teores foliares de $\mathrm{Ca}$ e $\mathrm{Mg}$ reduziram-se linearmente com o aumento das doses de $\mathrm{K}$ aplicadas ao solo. Essas reduções são atribuídas ao efeito competitivo entre esses nutrientes com o K (Quaggio et al., 2011). Os valores encontrados dos teores de $\mathrm{Ca}$ variaram de 36 a 44,36 a 43 e 33 a $38 \mathrm{~g} \mathrm{~kg}^{-1}$, e os de $\mathrm{Mg}$ variaram de 2,2 a 2,8, 2,9 a 3,3 e 3,0 a 3,3, na primeira, terceira e quinta safra, respectivamente, nas doses de $\mathrm{K}_{2} \mathrm{O}$ que proporcionaram a máxima eficiência econômica e onde não se aplicou $\mathrm{K}_{2} \mathrm{O}$. Esses valores foram próximos dos recomendados para o bom crescimento dos citros (Quaggio et al., 1996). No entanto, a aplicação de $200 \mathrm{~kg} \mathrm{ha}^{-1}$ de $\mathrm{K}_{2} \mathrm{O}$ reduziu os teores foliares de $\mathrm{Ca}$ e $\mathrm{Mg}$ de limão 'Tahiti' para 28 e $1,6,29$ e 2,5 e 28 e $2,7 \mathrm{~g} \mathrm{~kg}^{-1}$, na primeira, terceira e quinta safra, respectivamente, valores considerados abaixo dos níveis recomendados. As reduções dos teores de $\mathrm{Ca}$ e $\mathrm{Mg}$ foliar foram acompanhadas pela redução da produção em 24,37 e $23 \%$, na primeira, terceira e quinta safra, respectivamente. O excesso de $\mathrm{K}$ pode determinar perdas de produção dos citros, em razão do desequilíbrio nutricional, marcado pelo decréscimo acentuado dos teores foliares de $\mathrm{Ca}$ e $\mathrm{Mg}$ (Quagio et al., 2011). Por esse motivo, há a necessidade do monitoramento periódico do estado nutricional do pomar.

\section{Conclusões}

1. As doses de $\mathrm{N}$ para alcançar a máxima eficiência técnica de limão são 90, 99, 69, 66 e $100 \mathrm{~kg} \mathrm{ha}^{-1}$ de N e, para alcançar a máxima eficiência econômica, são $85,95,67,64$ e $93 \mathrm{~kg} \mathrm{ha}^{-1}$ de $\mathrm{N}$, na primeira, segunda, terceira, quarta e quinta safra, respectivamente.

2. As doses de $\mathrm{N}$ estimadas para alcançar a máxima eficiência econômica, na primeira e segunda safras, são muito superiores aos recomendados pela literatura.

3. As doses de $\mathrm{K}$ estimadas para alcançar a máxima eficiência técnica de limão são 126, 116, 101, 98 e $95 \mathrm{~kg} \mathrm{ha}^{-1}$ de $\mathrm{K}_{2} \mathrm{O}$, e as doses para obter a máxima eficiência econômica são $112,105,94,90$ e $88 \mathrm{~kg} \mathrm{ha}^{-1}$ de $\mathrm{K}_{2} \mathrm{O}$, na primeira, segunda, terceira, quarta e quinta safra, respectivamente.

4. As doses de $\mathrm{K}$ estimadas para obter a máximas eficiência econômica, nas cinco safras, são superiores às recomendadas pela literatura.

5. Os níveis críticos médios do $\mathrm{N}$ e $\mathrm{K}$ estimados nas folhas do limoeiro 'Tahiti' são 25 e $22 \mathrm{~g} \mathrm{~kg}^{-1}$, respectivamente; o nível crítico de $\mathrm{K}$ é superior ao descrito na literatura.

\section{Agradecimento}

À Fundação de Amparo à Pesquisa do Estado de Minas Gerais (Fapemig), pelo apoio financeiro (processo CAG-APQ-00561-12) e pela concessão de bolsa de incentivo a pesquisa.

\section{Referências}

ASSOCIAÇÃO CENTRAL DOS FRUTICULTORES DO NORTE DE MINAS. Cotação de frutas: limão para mercado interno. Janaúba: Abanorte, 2015. Disponível em: <http:// www.abanorte.com.br/producao/abanorte/cotacao-de frutas/ cotacoes_view?index_cotacao $=$ ec $65 \mathrm{~d} 59 \mathrm{~b} 9 \mathrm{bbef} 97 \mathrm{cff} 2 \mathrm{bc} 5 \mathrm{~d} 765 \mathrm{~d}$ 8e4ef\&tipo_ cotacao=Lim\%C3\%A3o\%20-\%20Taiti\%20-\%20 Exporta\% $\%$ C3\%A7\%C3\%A3o>. Acesso em: set. 2015.

ALVA, A.K.; MATTOS JR., D.; PARAMASIVAM, S.; PATIL, B.; DOU, H.; SAJWAN, K.S. Potassium management for optimizing citrus production and quality. International Journal of Fruit Science, v.6, p.3-43, 2006. DOI: 10.1300/J492v06n01_02.

ALVAREZ V., V.H. Avaliação da fertilidade do solo: superfície de resposta - modelos aproximativos pra expressar a relação fator-resposta. Viçosa: UFV, Imprensa Universitária, 1994. 75p.

AULER, P.A.M.; FIORI-TUTIDA, A.C.G.; TAZIMA, Z.H. Comportamento da laranjeira 'Valência' sobre seis porta-enxertos no noroeste do Paraná. Revista Brasileira de Fruticultura, v.30, p.229-234, 2008. DOI: 10.1590/S0100-29452008000100042.

CANTARELLA, H. Nitrogênio. In: NOVAIS, R.F. de; ALVAREZ V., V.H.; BARROS, N.F. de; FONTES, R.LF.; CANTARUTTI, R.B.; NEVES, J.C.L. (Ed.). Fertilidade do solo. Viçosa: Sociedade Brasileira de Ciência do Solo, 2007. p.375-470.

DIAS, J.R.M.; TUCCI, C.A.F.; WADT, P.G.S.; SILVA, A.M. da; SANTOS, J.Z.L. Níveis críticos e faixas de suficiência nutricional em laranjeira-pêra na Amazônia Central obtidas pelo método DRIS. Acta Amazonica, v.43, p.239-246, 2013. DOI: 10.1590/ S0044-59672013000300001.

DUARTE, I.N.; PEREIRA, H.S.; KORNDÖRFER, G.H. Lixiviação de potássio proveniente do termopotássio. Pesquisa Agropecuária Tropical, v.43, p.195-200, 2013. DOI: 10.1590/ s1983-40632013000200003.

FIDALSKI, J.; AULER, P.A.M. Adubação nitrogenada e precipitação sobre a nutrição e produção de laranjeira 'Valência'. 
Pesquisa Agropecuária Brasileira, v.42, p.141-146, 2007. DOI: 10.1590/S0100-204X2007000200001.

MAGALHÃES FILHO, J.R.; AMARAL, L.R. do; MACHADO, D.F.S.P.; MEDINA, C.L.; MACHADO, E.C. Deficiência hídrica, trocas gasosas e crescimento de raízes em laranjeira 'Valência' sobre dois tipos de porta-enxerto. Bragantia, v.67, p.75-82, 2008. DOI: 10.1590/S0006-87052008000100009.

MALAVOLTA, E.; VITTI, G.C.; OLIVEIRA, S.A. de. Avaliação do estado nutricional das plantas: princípios e aplicações. 2.ed. Piracicaba: Potafos, 1997. 319p.

MATTOS JUNIOR, D.; MILANEZE, T.F.; AZEVEDO, F.A.; QUAGGIO, J.A. Soil nutrient availability and its impact on fruit quality of 'Tahiti' acid lime. Revista Brasileira de Fruticultura, v.32, p.335-342, 2010.

MENDES, W. da C.; ALVES JUNIOR, J.; CUNHA, P.C.R. da; SILVA, A.R. da; EVANGELISTA; A.W.P.; CASAROLI, D. Lixiviação de nitrato em função de lâminas de irrigação em solos argiloso e arenoso. Irriga, v.1, p.47-56, 2015. DOI: 10.15809/ irriga.2015v1n2p47.

NEVES, C.S.V.J.; MURATA, I.M.; STENZEL, N.M.C.; MEDINA, C. de C.; BORGES, A.V.; OKUMOTO, S.H; LEE, R.H.C; KANAI, H.T. Root distribution of rootstocks for 'Tahiti' lime. Scientia Agricola, v.61, p.94-99, 2004. DOI: 10.1590/ S0103-90162004000100016.

OLIVEIRA, C.V.; KER, J.C.; CURI, N.; DUARTE, M.N.; FONTES, L.E.F. Caracterização de concreções ferro-manganosas de solos de calcário no norte do Estado de Minas Gerais. Revista Brasileira de Ciência do Solo, v 25, p.565-574, 2001. DOI: 10.1590/ S0100-06832001000300005.

PENNA, L.D.; AROUCA, M.B.; PRADO, R.M.; ROZANE, D.E.; CAMARA, H. Crescimento de mudas de lima ácida 'Tahiti', enxertadas em 'flying dragon' em função da fertirrigação com nitrogênio, fósforo e potássio. Nucleus, v.9, p.17-26, 2012. DOI: 10.3738/1982.2278.568.

QUAGGIO, J.A.; MATTOS JUNIOR, D.; BOARETTO, R.M. Sources and rates of potassium for sweet orange production. Scientia Agricola, v.68, p.369-375, 2011. DOI: 10.1590/ S0103-90162011000300015.

QUAGGIO, J.A.; RAIJ, B. van; PIZA Jr., C. de T. Frutíferas. In: RAIJ, B. van; CANTARELLA, H.; QUAGGIO, J.A.; FURLANI, A. M. C. (Ed.). Recomendações de adubação e calagem para o Estado de São Paulo. 2.ed. Campinas: Instituto Agronômico e Fundação IAC, 1996. p.121-153. (IAC. Boletim técnico, 100).

SANTANA, J. das G.; LEANDRO, W.M.; NAVES, R.V.; CUNHA, P.P. da. Normas DRIS para interpretação de análises de folha e solo, em laranjeira pêra, na região central de Goiás. Pesquisa Agropecuária Tropical, v.38, p.109-117, 2008.

SISTEMA brasileiro de classificação de solos. Brasília: Embrapa Produção de Informação; Rio de Janeiro: Embrapa Solos, 1999. $412 \mathrm{p}$.

SOUZA, L.D.; SOUZA, L. da S.; LEDO, C.A. da S. Sistema radicular dos citros em Neossolo Quartzarênico dos Tabuleiros Costeiros sob irrigação e sequeiro. Pesquisa Agropecuária Brasileira, v.42, p.1373-1381, 2007. DOI: 10.1590/ S0100-204X2007001000002.

SOUZA, L.H.; NOVAIS, R.F.; ALVAREZ V., V. H; VILLANI, E.M. de A. Efeito do $\mathrm{pH}$ do solo rizosférico e não rizosférico de plantas de soja inoculadas com Bradyrhizobium japonicum na absorção de boro, cobre, ferro, manganês e zinco. Revista Brasileira de Ciência do Solo, v.34, p.1641-1652, 2010.

TESTEZLAF, R.; COLETTI, C.; MATSURA, E.E. Distribuição de raízes da laranja 'Valência' irrigada por autopropelido. Pesquisa Agropecuária Brasileira, v.42, p.905-908, 2007. DOI: 10.1590/ S0100-204X2007000600020.

Recebido em 10 de setembro de 2015 e aprovado em 14 de março de 2016 